\title{
Imaging Electrode Heterogeneity Using Chemically Confined Fluorescence Electrochemical Microscopy (CCFEM)
}

Jiratheep Pruchyathamkorn, Minjun Yang, Hatem M.A. Amin, Christopher BatchelorMcAuley and Richard G. Compton*

* corresponding author: Richard G. Compton, Department of Chemistry, Physical \& Theoretical Chemistry Laboratory, University of Oxford, South Parks Road, Oxford, OX1 3QZ, United Kingdom

Email: richard.compton@chem.ox.ac.uk. Tel: +44(0)1865275957 Fax: +44(0)1865275410

\section{Abstract}

By varying the total and the relative concentrations of a strong acid $\left(\mathrm{HClO}_{4}\right)$ and a $\mathrm{pH}$ sensitive fluorescent dye (8-hydroxypyrene-1,3,6-trisulfonate), this work demonstrates that both the hydrogen evolution reaction (HER) or the oxygen reduction reaction (ORR) can be selectively and optically studied at an electrochemical interface. The local $\mathrm{pH}$ shift driven by the redox reaction can be visualised through fluorescence imaging of the interface. The use of finite strong acid concentrations further serves to constrain the $\mathrm{pH}$ change to a thin-layer adjacent to the surface. This chemical confinement of the fluorophore improves the systems resolution and enables micron scale heterogeneity on the electrode surface to be readily visualised.

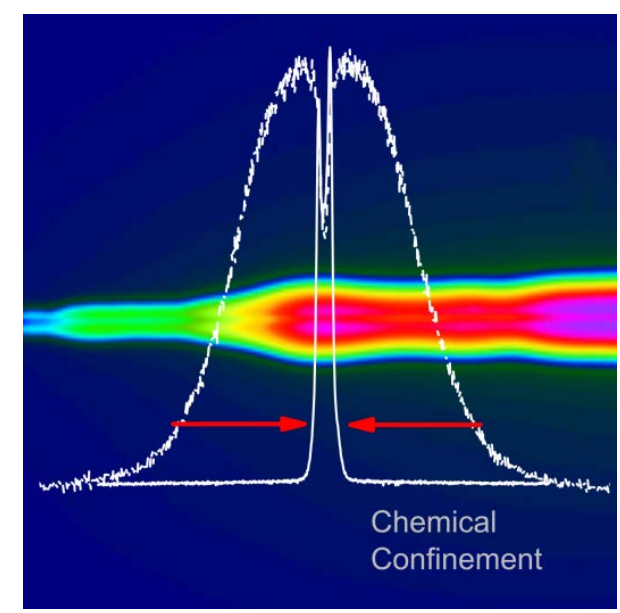


Interfacial charge transfer underpins many energy technologies notably batteries, supercapacitors and fuel cells. ${ }^{1-2}$ Although techniques such as voltammetry can yield significant thermodynamic and kinetic information regarding these systems, a major deficiency is the lack of spatial information. Insights may be inferred by studying processes at variable scan rates or by studying reactions at individual nanoparticles. ${ }^{3-4}$ However, although useful, such approaches are not always experimentally applicable. Since the mid80s the dominant route to address such questions of spatial heterogeneity has been through the use of scanning electrochemical microscopy (SECM) ${ }^{5-6}$ By combination of this technique with a variety of methods for controlling the tip-to-sample distance this approach has been advanced to a high state-of-art. ${ }^{7-9}$ However, as a technique SECM is still very regularly employed using probe electrodes of micron dimensions. ${ }^{10}$ Moreover, although widely applied, such methodologies are still not without limitations. Ultimately, the imaging time is limited by the rate at which the systems can raster across the surface so as to acquire an image. First, obtaining information about system dynamics is challenging. Second, diffusional blurring of the solution phase electroactive species often limits the systems resolution. Third, issues relating to solution phase convection can occur on these time scales with associated image loss. ${ }^{11-12}$

Fluorescence Electrochemical Microscopy (FEM) is an alternate technique for imaging electrode heterogeneity. ${ }^{13-14}$ The use of FEM has been far less studied in the literature than SECM; this is in spite of the significant advantage of sampling electrode areas in parallel and at video frequencies. A major limitation for the technique arises from diffusional blurring leading to the relatively rapid transport of the fluorophore away from an active site. In SECM these issues are partially minimised by the probe consuming the electrode product. Recent work has looked to use confocal microscopy to improve the applicability of FEM systems; ${ }^{15}$ however, such approaches are again limited by the need to form images by rastering. In contrast, Zhang et al. demonstrated in their use of a bipolar electrode that a coupled homogeneous reaction can be used to constrain a fluorophore to a smaller volume. ${ }^{16}$ Work has built on this result and showed that by constricting a fluorophore to a 'thin' reaction-layer the spatial resolution of the imaging systems can be dramatically improved. ${ }^{17}$

In the biological sciences fluorophores that are sensitive to a variety of specific reagents have been developed. ${ }^{18}$ However, due to the near ubiquity of proton-coupled electron transfers $(\mathrm{PCET})^{19}$ this work will focus on providing and evidencing the applicability of a more 
general methodology for visualising local $\mathrm{pH}$ changes at an electrochemical interface and hence indirectly monitoring the course of an interfacial reaction. A variety of techniques and methodologies have been developed to probe such local $\mathrm{pH}$ changes during the course of interfacial reactions. ${ }^{20-21}$ In this work, the technique of Chemically Confined Fluorescence Electrochemical Microscopy (CCFEM) is employed to image heterogeneity in electrode activity. Specifically, the hydrogen evolution reaction (HER) and the oxygen reduction reaction (ORR) are imaged at an electrochemical interface as a function of the applied potential.

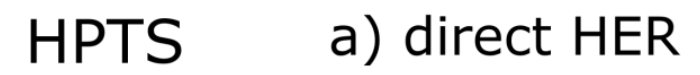

b) PCET

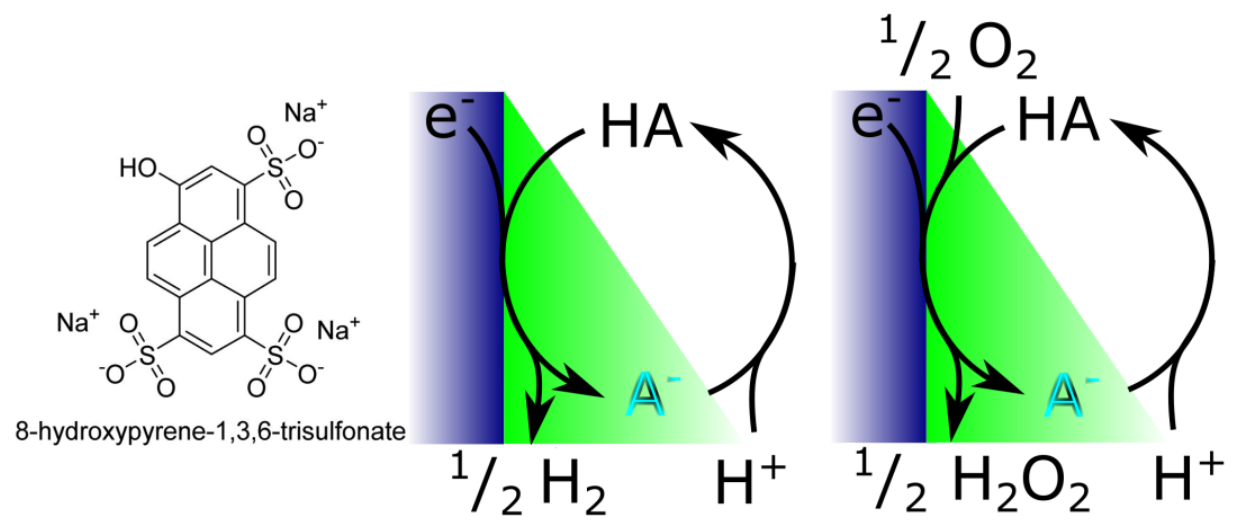

Scheme 1: The structure of the used fluorophore HPTS and the two main routes by which the $\mathrm{pH}$ at an electrochemical interface can be increased due to the occurrence of a reduction process. The blue area represents the electrode and the green schematically represents the fluorescence intensity as a function of the distance perpendicular to the electrode surface. $H A$ represents a weak acid fluorophore that in this case exhibits strong fluorescence in its deprotonated form $\left(A^{-}\right)$, in the present work HPTS is used. a) HER leads to the consumption of protons $b)$ PCET, exemplified here as the reduction of oxygen, leads to proton consumption. In the latter case it is necessary that the $p K_{a}$ of the product of the PCET reaction has a $p K_{a}$ above that of the fluorogenic $\mathrm{pH}$ indicator (HA). In both cases the presence of a low concentration of a strong acid $\left(\mathrm{H}^{+}\right)$in bulk solution ensures the spatial extent of the deprotonated species $A^{-}$is restricted to a volume close to the electrode surface.

The fluorophore 8-hydroxypyrene-1,3,6-trisulfonate (HPTS, Scheme 1) provides an effective route for monitoring local $\mathrm{pH}$ changes using optical fluorescence microscopy. The phenol group has an associated $\mathrm{pK}_{\mathrm{a}}$ of 7.21 and deprotonation shifts the absorption maxima from 403 $\mathrm{nm}$ to $454 \mathrm{~nm} .{ }^{17}$ This change in the absorption spectra facilitates visualisation of alkaline regions of solution. Apart from being a proton source, this fluorescent dye does not exhibit any reductive voltammetric response in the aqueous potential window. ${ }^{17}$ Electrochemical 
redox reactions may drive $\mathrm{pH}$ changes at an electrode interface either directly via the $\mathrm{H}^{+} / \mathrm{H}_{2}$ redox couple or more broadly as a result of the occurrence of a PCET reaction. This work starts by visualising the HER at a carbon fibre electrode.

A general strategy for the visualisation of the HER is shown in Scheme 1; also presented is a scheme for the visualisation of PCET reactions (vide infra). In the first case the direct reduction of protons at the electrochemical interface results in a shift in the $\mathrm{pH}$ local to the surface. Depending on the $\mathrm{pH}$ environment and electrode potential the HER may precede either via the reduction of hydronium ions and/or protons bound to a conjugate base. At an unmodified carbon fibre electrode the reduction of perchloric acid $\left(1.0 \mathrm{mM} \mathrm{HClO}_{4}\right.$ in $0.1 \mathrm{M}$ $\mathrm{KClO}_{4}$ and containing $0.5 \mathrm{mM}$ HPTS) occurs at $-1.5 \mathrm{~V}$ vs Ag (See SI section 1). In contrast solvent break down (in this case HER from water) does not occur until -1.75 V vs Ag. Figure 1 shows a series of fluorescence microscope images of a $7 \mu \mathrm{m}$ diameter carbon fibre wire in an aqueous solution containing $0.1 \mathrm{M} \mathrm{KClO}_{4}, 0.5 \mathrm{mM}$ HPTS and between 0.55-1.00 mM $\mathrm{HClO}_{4}$. The electrode potential was swept from 0.2 to $-1.8 \mathrm{~V}$ vs Ag at a scan rate of $0.1 \mathrm{~V} \mathrm{~s}^{-1}$. During the course of the scan the wire was imaged optically using an excitation wavelength of $475 \pm 17.5 \mathrm{~nm}$ and an emission filter at $530 \pm 21.5 \mathrm{~nm}$. Images were recorded at 10 f.p.s using a monochrome camera (16-bit, 4 megapixel image), the resulting grey scale images have been false coloured to clearly indicate the change in image intensity. Figure 1 presents still images of the wire at select potentials during the course of the electrochemical scan.

At higher overpotentials proton reduction leads to an increase in the solution phase $\mathrm{pH}$ resulting in an increase in the fluorescence intensity in these local low proton concentration environments. As can be seen in Figure 1 and further exemplified in the SI section 2, the potential at which a significant change in $\mathrm{pH}$ and hence an increase in fluorescence occurs varies as a function of the acid concentration. At lower acid concentrations the exhaustion of the local proton concentration occurs at a less negative potential resulting in the earlier onset of the fluorescence signal. It should be highlighted that in the above experiments due to the requirement of using a thin layer cell the removal of oxygen from the electrode systems is not feasible; consequently, with the use of lower acid concentrations, as shown in SI section 2, the onset of the fluorescence occurs at a lower potential partly due to the consumption of protons by the ORR. The onset of the fluorescence signal is sensitive to the net proton consumption at the electrode surface relative to the solutions buffering capacity, as a consequence of this, the ability to selectively image the ORR is discussed further below. 


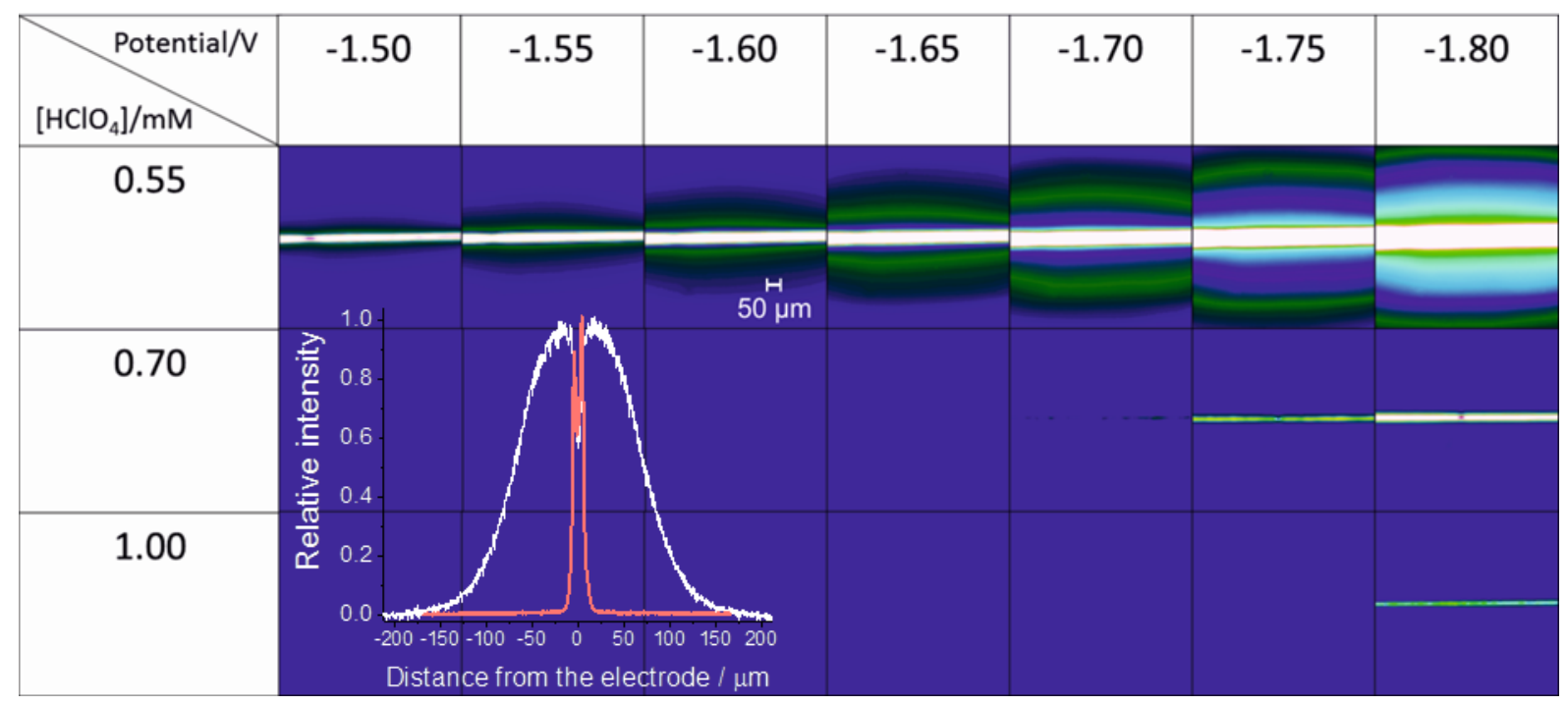

Figure 1: Fluorescence microscope images for carbon microfibre (diameter $7 \mu \mathrm{m}$ ) with variable concentration of perchloric acid (in $0.1 \mathrm{M} \mathrm{KClO}_{4}$ and $0.5 \mathrm{mM} \mathrm{HPTS}$ ). Data acquired at 10 f.p.s. during the course of a voltammetric scan from 0.2 to $-1.8 \mathrm{~V}$ at $0.1 \mathrm{~V} \mathrm{~s}^{-1}$ (potentials reported against a pseudo Ag reference), images at select potentials have been reproduced. Scaling is the same for all images and have been false coloured to emphasis the variation in fluorescence intensity (see Figure S5). Colours vary from dark blue to white (low to high fluorescence intensity). Inlay depicts the cross sectional intensity profile across the carbon fibre wire at $-1.8 \mathrm{~V}$ for 0.55 (white) and 1.0 (red) $\mathrm{mM} \mathrm{HClO4}$, clearly demonstrating the confinement of the fluorophore in the higher acid conditions.

The HER shown in Figure 1 demonstrates, first, for this unmodified carbon fibre electrode the fluorescence signal is observed to be predominantly homogeneous along the length of the wire. Second, in all cases at higher overpotentials the rate of proton consumption at the electrode surface overcomes that of the diffusion from the bulk solution, resulting in an increase in the intensity and spatial extent of the fluorescence signal. Importantly, at low overpotentials the spatial extent of this fluorescence signal is contracted as compared to the diffusion length scale. ${ }^{22}$ This contraction occurs due to the presence of the bulk proton concentration which ensures the dimensions of the produced alkaline region are confined and results in a length scale less than the diameter of the electrode $(7 \mu \mathrm{m})$ as evidenced by the optical images. To demonstrate the benefits of this chemically confined fluorescence signal the HER was studied at an epoxy-sized carbon fibre electrode (i.e. a wire coated with a thin layer of epoxy). SI Section 3 depicts representative images of the fibre as a function of the applied potential. In contrast to the earlier result (Figure 1) for the unmodified carbon surface the electrode demonstrates significant heterogeneity. Partial physical blocking of the 
electrode surface by the epoxy leads to heterogeneous activity of the electrode, with activity likely at defects along the surface.

Although of distinct physical interest, in terms of applications imaging the HER is limited in scope. However, a diverse number of redox reactions are coupled with proton transfer, ORR being a paradigmatic example. Depending on the material and electrode potential this reaction may either proceed via a 4 or 2 electron reduction pathway. Due to the associated proton consumption, under low buffering conditions the reaction can cause a $\mathrm{pH}$ change at the electrode interface. The solubility of oxygen in water at $25^{\circ} \mathrm{C}$ is $1.24 \mathrm{mM},{ }^{23}$ consequently under atmospheric conditions a non-degassed solution will contain $\sim 0.27 \mathrm{mM}$ oxygen. In order for the ORR to be visualised by a $\mathrm{pH}$ change it is imperative that the availability of protons is sufficiently low that the reaction will drive a local increase in alkalinity. Figure 2 depicts the response of a platinum nanoparticle modified carbon fibre electrode and as a comparison an unmodified section of the same wire. The carbon fibre wire electrode was modified with platinum nanoparticles (diameter $=50 \mathrm{~nm}$, nanoComposix, CA USA) by dropcasting from a stock solution (55 pM), following which the electrode was rinsed with distilled water. SI section 4 presents SEM images of a carbon fibre wire modified following this procedure. To allow direct comparison the fluorescence image of the carbon fibre modified with platinum nanoparticles is situated at the edge of the modified and unmodified sections (the position of which is indicated in the Figure 2). For further comparison a full image of an unmodified section of wire is also depicted in Figure 2. In this experiment the solution contained $0.1 \mathrm{M} \mathrm{KClO}_{4}, 0.1 \mathrm{mM} \mathrm{HClO}_{4}$ and $0.1 \mathrm{mM}$ HPTS and the electrode potential was scanned reductively at $0.1 \mathrm{~V} \mathrm{~s}^{-1}$ from 0.2 to $-1.2 \mathrm{~V}$ (data shown to $-0.9 \mathrm{~V}$ ). Due to the lower total proton concentration the increase in the fluorescence signal occurs at a significantly lower electrode potential as compared to the results presented in Figure 1. The activity of the platinum modified section is seen to be significantly greater than that of the bare carbon. The initial onset for the fluorescence response on the platinum nanoparticle modified section of the wire occurs at a potential of -0.3 to $-0.4 \mathrm{~V}$ (vs Ag) as compared to 0.6 to $-0.7 \mathrm{~V}$ (vs Ag) for the bare carbon surface. 


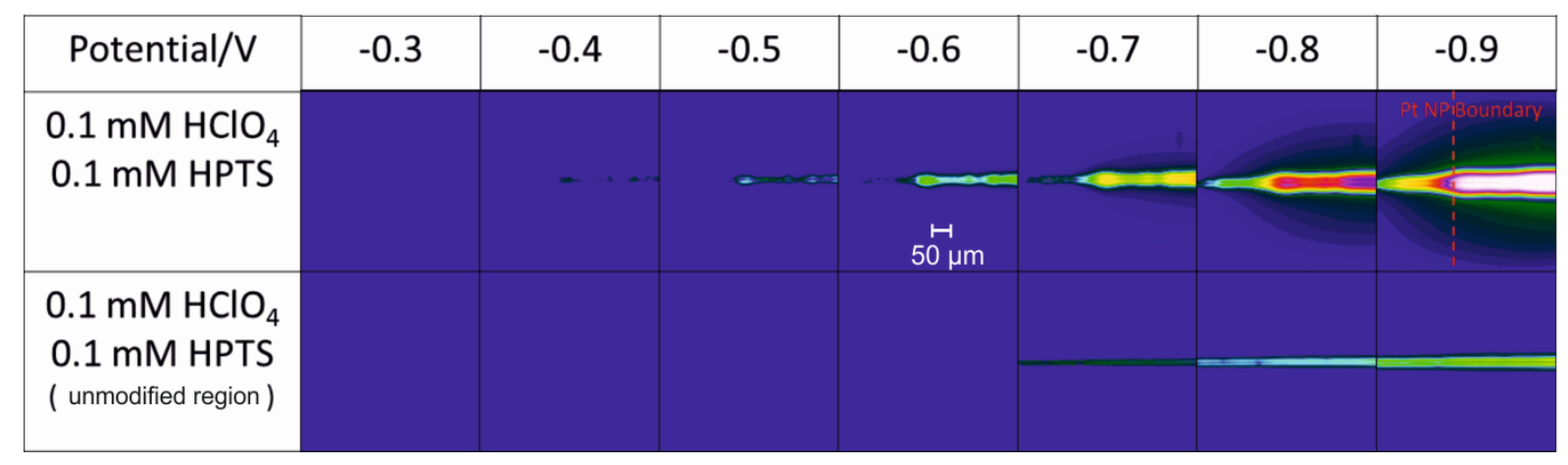

Figure 2: Fluorescence microscope images for 40 minutes platinum nanoparticle coated carbon microfibre (diameter $7 \mu \mathrm{m}$ ), $0.1 \mathrm{mM} \mathrm{HClO}_{4}, 0.1 \mathrm{mM} \mathrm{HPTS}$ and $0.1 \mathrm{M} \mathrm{KClO}$. Data acquired at 10 f.p.s. during the course of a voltammetric scan from 0.2 to $-0.9 \mathrm{~V}$ (potentials reported against a pseudo Ag reference), images at select potentials have been reproduced. Images are of the same wire but focussed on different Pt modified and un-modified areas. Images have been false coloured (see Figure S5), to emphasise the variation in fluorescence intensity. Colours vary from dark blue to white (low to high fluorescence intensity).

By chemically constraining the fluorophore in the solution phase to a restricted volume adjacent to an electrochemical interface, CCFEM can provide information regarding localised electrode activity. The systems sensitivity towards a given redox process can be optimised by varying the ratio and total concentration of the reagents used. In this manner it has been demonstrated that by using 'high' proton concentrations (in this work [HPTS] $+\left[\mathrm{HClO}_{4}\right]$ $>1.0 \mathrm{mM}$ ) the HER reaction can be studied. By lowering the total acid concentration to 0.2 $\mathrm{mM}$ the ORR can be investigated and the difference in activity of two electrode materials (platinum and carbon) can be easily visualised as a function of the electrode potential. The ability of CCFEM to image large areas at video frequencies provides a number of advantages; the system more readily lends itself to studying dynamic processes and for use in high through-put applications. The approach presented herein is general; however, application to other systems, although far from insurmountable, will require some chemical ingenuity

\section{Acknowledgements}

The research leading to these results has received funding from the European Research Council under the European Union's Seventh Framework Program (FP/2007-2013)/ERC Grant Agreement No. 320403.

\section{Supporting Information}


Electrochemical response of a carbon fibre electrode for the ORR and HER. Fluorescence imaging of carbon fibre as a function of acid concentration. Imaging the HER at an epoxy coated carbon fibre. SEM images of a carbon fibre modified with PtNPs. Experimental details and optical cell design.

\section{References}

1. Aricò, A. S.; Bruce, P.; Scrosati, B.; Tarascon, J. M.; Van Schalkwijk, W. Nanostructured Materials for Advanced Energy Conversion and Storage Devices. Nat. Mat. 2005, 4 (5), 366-377.

2. Steele, B. C. H.; Heinzel, A. Materials for Fuel-Cell Technologies. Nature 2001, 414 (6861), 345-352.

3. Batchelor-McAuley, C.; Kätelhön, E.; Barnes, E. O.; Compton, R. G.; Laborda, E.; Molina, A. Recent Advances in Voltammetry. ChemistryOpen 2015, 4 (3), 224-260.

4. Stevenson, K. J.; Tschulik, K. A Materials Driven Approach for Understanding Single Entity Nano Impact Electrochemistry. Curr. Opin. Electrochem. 2017.

5. $\quad$ Engstrom, R. C.; Weber, M.; Wunder, D. J.; Burgess, R.; Winquist, S. Measurements within the Diffusion Layer Using a Microelectrode Probe. Anal. Chem. 1986, 58 (4), 844-848.

6. Kwak, J.; Bard, A. J. Scanning Electrochemical Microscopy. Theory of the Feedback Mode. anal. Chem 1989, 61 (11), 1221-1227.

7. Hengstenberg, A.; Kranz, C.; Schuhmann, W. Facilitated Tip-Positioning and Applications of Non-Electrode Tips in Scanning Electrochemical Microscopy Using a Shear Force Based ConstantDistance Mode. Chem. Eur. J. 2000, 6 (9), 1547-1554.

8. Ebejer, N.; Schnippering, M.; Colburn, A. W.; Edwards, M. A.; Unwin, P. R. Localized High Resolution Electrochemistry and Multifunctional Imaging: Scanning Electrochemical Cell Microscopy. Anal. Chem. 2010, 82 (22), 9141-9145.

9. Wain, A. J.; Pollard, A. J.; Richter, C. High-Resolution Electrochemical and Topographical Imaging Using Batch-Fabricated Cantilever Probes. Anal. Chem. 2014, 86 (10), 5143-5149.

10. Ventosa, E.; Madej, E.; Zampardi, G.; Mei, B.; Weide, P.; Antoni, H.; La Mantia, F.; Muhler, M.; Schuhmann, W. Solid Electrolyte Interphase (Sei) at Tio $<\operatorname{Inf}>2</$ Inf $>$ Electrodes in Li-lon Batteries: Defining Apparent and Effective Sei Based on Evidence from X-Ay Photoemission Spectroscopy and Scanning Electrochemical Microscopy. ACS Appl. Mat. Int. 2017, 9 (3), 3123-3130.

11. Novev, J. K.; Compton, R. G. Thermal Convection in Electrochemical Cells. Boundaries with Heterogeneous Thermal Conductivity and Implications for Scanning Electrochemical Microscopy. Phys. Chem. Chem. Phys. 2017, 19 (20), 12759-12775.

12. Novev, J. K.; Compton, R. G. Natural Convection Effects in Electrochemical Systems. Curr. Opin. Electrochem. 2017.

13. Bowyer, W. J.; Xie, J.; Engstrom, R. C. Fluorescence Imaging of the Heterogeneous Reduction of Oxygen. Anal. Chem. 1996, 68 (13), 2005-2009.

14. Engstrom, R. C.; Ghaffari, S.; Qu, H. Fluorescence Imaging of Electrode-Solution Interfacial Processes. Anal. Chem. 1992, 64 (21), 2525-2529.

15. Bouffier, L.; Doneux, T. Coupling Electrochemistry with in Situ Fluorescence (Confocal) Microscopy. Curr. Opin. Electrochem. 2017.

16. Guerrette, J. P.; Percival, S. J.; Zhang, B. Fluorescence Coupling for Direct Imaging of Electrocatalytic Heterogeneity. J. Am. Chem. Soc. 2012, 135 (2), 855-861.

17. Yang, M.; Batchelor-McAuley, C.; Kätelhön, E.; Compton, R. G. Reaction Layer Imaging Using Fluorescence Electrochemical Microscopy. Anal. Chem. 2017.

18. Lemke, E. A.; Schultz, C. Principles for Designing Fluorescent Sensors and Reporters. Nat. Chem. Bio. 2011, 7 (8), 480-483. 
19. Huynh, M. H. V.; Meyer, T. J. Proton-Coupled Electron Transfer. Chem. Rev. 2007, 107 (11), 5004-5064.

20. Singh, M. R.; Kwon, Y.; Lum, Y.; Ager, J. W.; Bell, A. T. Hydrolysis of Electrolyte Cations Enhances the Electrochemical Reduction of $\mathrm{Co}<\operatorname{lnf}>2</ \operatorname{Inf}>$ over Ag and Cu. J. Am. Chem. Soc. 2016, 138 (39), 13006-13012.

21. Ayemoba, O.; Cuesta, A. Spectroscopic Evidence of Size-Dependent Buffering of Interfacial Ph by Cation Hydrolysis During Co<Inf $>2</$ Inf $>$ Electroreduction. ACS Appl. Mat. Int. 2017, 9 (33), 27377-27382.

22. 10 microns, $\delta \sim(2 D t)^{\wedge} 0.5$, where $D$ is the diffusion coefficient $\left(m^{\wedge} 2 \mathrm{~s}-1\right)$ and $t$ is the experimental time (s)

23. Battino, R.; Rettich, T. R.; Tominaga, T. The Solubility of Oxygen and Ozone in Liquids. J. Phys. Chem. Ref. Data 1983, 12 (2), 163-178. 